Prepared by Justine MacNeil, MD, University of Utah Wilderness Medicine Fellow, Salt Lake City, UT, USA.

\section{IEEE TRANSACTIONS ON CYBERNETICS}

\section{A Multirobot Path-Planning Strategy for Autonomous Wilderness Search and Rescue}

Mobile robotics has played an increasingly important role in search and rescue efforts. The success of robotics in urban search and rescue environments has encouraged its expansion to wilderness search and rescue. Wilderness search and rescue presents unique challenges, including less predictable terrain, a moving target, and an increasing search area as time progresses. Current wilderness search and rescue groups primarily use human searchers; however, the authors believe that multirobot coordination results in more systematic coverage of the search area. This paper proposes a novel path-planning strategy for autonomous multirobot coordination in wilderness search and rescue scenarios.

The investigators identify 3 important components when using multirobot coordination for wilderness search and rescue: planning initial paths, implementing and evaluating the paths, and replanning the paths if necessary. When creating a path to be followed by the robots, the investigators propose a novel process that creates boundaries using "iso-probability curves." These curves are constructed based on rays from the last known point of the lost human subject. Multiple autonomously functioning robots that communicate with each other will cover these points on the curves, in the hope of finding the lost subject. Although robots capable of performing this function still need to be fully implemented, human teams can also utilize the iso-probability curves to develop improved and systemic search methods. Even though there are recognized limitations, including difficult terrain, the researchers' proposal creates an effective model for robot path planning during wilderness search and rescue efforts.

(IEEE Trans Cybern. 2014 Nov 3 [Epub ahead of print]) A Macwan, J Vilela, G Nejat, B Benhabib.

Prepared by Matthew Stewart, MD, University of Utah Global Medicine Fellow, Salt Lake City, UT, USA.

\section{INTERNATIONAL JOURNAL OF ENVIRONMENTAL RESEARCH AND PUBLIC HEALTH}

The Causes and Circumstances of Drinking Water Incidents Impact Consumer Behaviour: Comparison of a Routine Versus a Natural Disaster Incident

A government must be able to effectively relay important public health information to its constituents. Informing the public is a difficult task: during Hurricane Rita, only $31 \%$ of the population was even aware of a "Boil Water" notice. This study compares the United Kingdom's public reaction to the same instruction under different circumstances. When municipal water sources become contaminated, water companies and local press both instruct the public on how to treat their water: Do Not Use, Do Not Drink, or Boil Water. This article compares public response to two different Boil Water notices, one after a routine incident in which a rabbit contaminated a treatment plant, and one after a natural disaster, namely, serious flooding in 2007 that resulted in a 7-day Do Not Drink notice before the Boil Water notice. The researchers analyzed consumers' compliance, use of information sources, recall of advice received, and satisfaction with information.

Data were collected by mailing surveys to 1000 households affected by the routine incident. Surveys were similar to those used to gather data after the 2007 floods. The researchers concluded that the routine incident resulted in greater use of water companies as information sources and greater compliance, as well as greater consumer satisfaction with information. After the floods, water companies and local radio were used an equal amount, but radio was found to be more useful. Noncompliance, defined as drinking water that was not boiled, was $5 \%$ after the routine incident and $29.3 \%$ after the floods. These observations may be explained by the water company's street campaigns to boil water, which was not possible during flooding.

A limitation of the study includes a low response rate for both surveys (below 20\%), making conclusions difficult to generalize. Additionally, surveys were administered 8 months after the routine incident and 18 months after the natural disaster incident, possibly decreasing participant recall.

(Int J Environ Res Pub Health. 2014;11:11915-11930) G Rundblad, O Knapton, PR Hunter.

Prepared by August Longino, Research Assistant, University of Utah, Salt Lake City, UT, USA.

\section{PLOS ONE}

Design and Development of a Novel Vaccine for Protection Against Lyme Borreliosis

Lyme borreliosis is the most common vector-borne infection in the Northern Hemisphere, with an estimated 300,000 cases per year in the United States. Despite the high incidence and significant consequences, there is currently no vaccine available to humans. Vaccines have been attempted in the past using outer surface protein A (OspA) from Borrelia burgdorferi as the antigen stimulating the immune response. This is one of the dominant antigens expressed by $B$. burgdorferi when present in a tick. The species that causes Lyme disease in Europe varies from that found in the United States. The goal of this new vaccine is to target several pathogenic species through the development of a multivalent OspA vaccine in the hope of reducing the incidence of Lyme borreliosis.

To develop a vaccine, the researchers used the C-terminal end of the OspA protein because it is more distant from the bacterial surface and the majority of accessible epitopes have 
been localized there. The vaccine they produced included three proteins, each containing two OspA serotypes linked to a heterodimer. To preserve the vaccine's stability at physiologic temperatures, disulfide bonds were introduced. After the development of the vaccine, mice were immunized 3 times with $3 \mu \mathrm{g}$ vaccine. These mice were then challenged with, and shown to be protected against, infected ticks and in vitro grown spirochetes. Subsequent enzyme-linked immunosorbant assays induced a strong antibody response, confirming the protective potency of the vaccine.
Although this study was conducted in mice models, it lays the foundation for the generation of a vaccine against several Borrelia species. With future investigation, there is promise of a vaccine providing protection against Lyme disease in humans.

(PLoS ONE. 2014;9:1-12) P Comstedt, M Hanner, W Schüler, et al.

Prepared by Lindsey Caley, MD, University of Utah Wilderness Medicine Fellow, Salt Lake City, UT, USA. 